where unwarranted variation is evident and support the transition needed to build sustainable services for the future. The presentation will discuss how the framework can be applied within hospice and palliative care settings, to meet the needs of people near to or at the end of life to achieve the overarching ambition as agreed in partnership with National Voices and the National Council for Palliative Care; 'I can make the last stage of my life as good as possible because everyone works together confidently, honestly and consistently to help me and the people who are important to me, including my carer(s).'

\section{P-277 BEHAVE YOURSELF: IMPLEMENTING A BEHAVIOURS FRAMEWORK AS PART OF PERFORMANCE MANAGEMENT}

Robert Tuttle, Fiona David. Longfield, Gloucester, UK

10.1136/bmjspcare-2017-hospice.302

Background Staff are an organisation's biggest resource, largest investment and most significant asset. Encouraging staff to give their best is every organisation's greatest challenge. Managing performance is central to this. It is no longer sufficient for an organisation to focus on 'what' people do; they must also focus on 'how' staff are expected to do it. Encouraging exceptional behaviour through positive behaviour, attitude and approach encourages staff to recognise when they are at their best and a Behaviours Framework provides the structure that enables this.

Aim To design a Performance and Development Review (PDR) process with a Behaviours Framework at its core that links the organisation's core values with the exceptional behaviours and positive attitudes and approaches expected of all staff.

Methods Following a staff survey that highlighted concerns over how poor performance was managed, a review of multiorganisational performance management processes and behaviours frameworks was undertaken. Led jointly by HR and Education, an internal cross-organisational working group embarked on a six-month design and consultation period which concluded with intensive all staff training prior to implementation April 2017.

Results All staff are tasked with taking responsibility for the completion of their own PDR and having this in place by 30 June 2017. A quality assurance review will follow and a staff survey circulated asking for evaluative feedback. Any emerging issues will be assessed and addressed through further staff training.

Conclusion Longfield's new PDR and Behaviours Framework strikes a balance between managing the 'what' and the 'how' of staff performance. It provides staff and managers with a 'common language' and a framework with which to recognise exceptional and challenge unacceptable behaviour and attitude. It encourages a dialogue between staff and managers and celebration of the exceptional behaviour and attitude that Longfield demands of its most significant resource and asset - its staff!

\section{P-278 'WORKING TOGETHER BECAUSE WE CARE' - DEVELOPING OUR NEW TALENT AND PEOPLE STRATEGY}

Nicki Shaw, Carmel Millar, Julia Russell. Princess Alice Hospice, Esher, UK

10.1136/bmjspcare-2017-hospice.303

Background In June 2016 the Board approved an ambitious five-year strategy with the aim of 'Reaching more people'. The strategy depends on our ability to attract and retain dedicated, caring, skilled, high performing people across the hospice. We needed a Talent and People Strategy (T and PS) that would support our strategic aspirations.

Aims To develop a T and PS to ensure we have the right people with the right expertise, skills and values to meet our business needs both now and in five years' time.

Methods We engaged a senior Human Resources/Organisation Development practitioner to work with us to develop the strategy. We wanted them to use a rigorous and participative approach to:

- Consider what our workforce may look like in five years' time - including the potential to widen the role of volunteers

- Identify gaps in skills, expertise and competencies and plans to fill them

- Challenge pre- and/or mis-conceptions about structures, skill mix, role design, professional boundaries etc

- Consider talent management and succession planning so that we make best use of existing talent and capabilities.

Results $80 \%$ of our 406 paid staff (303 FTE) and many of our volunteers participated in a 'Fathoming the future' workshop, sharing ideas about what a future workplace might look like. The top ten priorities were shaped into five ambitions for our people that underpin the T and PS and provide the structure for a series of recommendations, actions and milestones for the next three years.

Conclusions By developing a T and PS in a structured and participative way we have a framework that enables us to make decisions around structures, skill mix, and succession planning and at the same time focus on the priorities that are most important to our staff, all in the context of an over-all plan which will best assure the success of our new strategy.

\section{P-279 USING CREATIVE PRACTICE DEVELOPMENT METHODOLOGIES TO CREATE A PERSON-CENTRED RECRUITMENT PROCESS}

${ }^{1}$ Melanie Legg, ${ }^{1}$ Anne Cleary, ${ }^{2}$ Caroline Dickson, ${ }^{2}$ Brendan McCormack. 'Marie Curie, London, UK; ${ }^{2}$ Queen Margaret University, Edinburgh, UK

10.1136/bmjspcare-2017-hospice.304

Background Marie Curie currently has 18 Practice Development Facilitators (PDFs) across the UK working in both hospice and community. The PDF role transitioned from the traditional Practice Educator role in August 2015, where the focus is now on facilitating learning and development opportunities rather than mandatory training. In partnership with Queen Margaret University the Practice Development Team 
have developed their knowledge and understanding of practice development methodologies and the impact this can have on developing person-centred cultures.

Aims Use creative practice development methodologies to bring about culture change where current staff engage with possible recruits and feedback into the recruitment process.

To ensure that potential new recruits fully understand the role on which they were embarking and experience these methods in practice during interview.

To improve retention of new staff to this role.

Methods The methods used were based on the Practice Development Workbook (Dewing, McCormack, \& Titchen, 2014). The interview was conducted in three parts; the first two exercises are group activities. Using creativity to understand their journey to here; a values exercise to explore their values as well as Marie Curie's and then a short individual interview. Results It has been challenging at times as the interview process is around three hours rather than the traditional style of interview of at least one hour. Feedback received from individuals who have completed this process have been highly evaluated.

Conclusion To build authentic and meaningful relationships and cultures it is important to identify individual values and beliefs (Wilson, 2011). Establishing person-centred cultures requires a sustained commitment from individuals and organisations. The focus on staff and service users is vital to ensuring compassionate care and a flourishing culture.

\section{P-280 TAPPING INTO THE BENEFITS OF THE ASSISTANT PRACTITIONER}

Hannah Ives. ellenor Gravesend, UK

10.1136/bmjspcare-2017-hospice.305

Background Nationally, the current shortage of nurses is likely to increase, in part due to the cessation of student nurse and other healthcare student bursaries (Imison \& Dayan, 2016). To address this workforce gap, organisations have re-evaluated the role of health care assistants (HCAs) and their potential to develop in the work place (Skills for Health, 2015). Assistant Practitioners work at Band 4, with increased knowledge and skills developed to enable them to assess, analyse and support care needs beyond the traditional role of healthcare assistants (Skills for Health, 2015). Yet little is known if, and how, the Assistant Practitioners would aid the staffing shortfall.

Aims - To identify benefits of Assistant Practitioner role in a hospice setting.

- To successfully embed an Assistant Practitioner within the team.

Methods - A combined approach was used to capture relevant information, including:

- Case studies around Assistant Practitioners

- Current career development pathways and guidelines for Assistant practitioners

- Job descriptions compared

- Questionnaire generated and conducted within a hospice

- Clear job description and pathways created.

Results Benefits of an Assistant Practitioner were widely recognised particularly: greater ability to support and assist care ; significance in increased work responsibilities; staff retention and career progression. Organisations adopted different ways to implement the Assistant Practitioner role demonstrating the diversity possible, including specialising in one area of work. Previous pitfalls centred on lack of communication about the role and responsibilities, acting as a barrier for staff acceptance. Our decision was appoint a new Assistant Practitioner with clear remit and responsibilities. Early benefits we are experiencing include:

- Supporting HCAs specifically with training and competencies

- Freeing specialist nurse time

- Bridging gap between staff grades.

Conclusion Assistant Practitioners can integrate into hospice teams, improving skill mix. Whilst the role continues to evolve, this generates exciting opportunities to develop and adapt work streams; early days but positive signs.

\section{P-281 DEVELOPING THE ROLE OF THE LYMPHOEDEMA ASSISTANT PRACTITIONER AT ST CATHERINE'S HOSPICE}

Ann McFarlane, Debbie Murphy. St Catherine's Hospice, Lancashire, UK

\subsection{6/bmjspcare-2017-hospice.306}

Background St Catherine's Hospice has supported the development of an assistant practitioner within its lymphoedema service as part of a workforce development strategy.

Aims To develop the role of the current support worker to address the skills shortage and recruitment difficulties within the specialty of lymphoedema

To free the specialist nurses to focus on more advanced work.

Methods To ensure the continued provision of high quality patient care, the retention and development of their workforce and taking into account the impact of current financial pressures, a workforce development analysis was done to identify where changes needed to be made. It was identified that the development of the current support worker into an assistant practitioner role was critical. Funding for the project was sought from the apprenticeship scheme and agreement with the senior management team. The education department facilitated the enrolment process with University of Central Lancashire.

Results The evaluation process has not been fully developed at this stage as the training is still in progress.

The following benefits have been identified:

- A clear well defined career pathway

- Specific set core competencies have been developed in line with Skills for Health; this has enhanced multi-disciplinary team working based on clarity of role responsibilities and levels of accountability

- Creating investment in staff training and development increases the recruitment and retention of staff therefore gives job satisfaction

- Individual personal development through increasing knowledge and confidence.

Conclusion The impact on the service has been evident in terms of staff absence for protected learning time as we are such a small team and backfill has proved to be difficult. A reduction in patient treatments as the support worker carries out the low-level interventions. Protected time for clinical learning has also been difficult at times and has been a strain on an already busy department. 